\title{
Best practice for infection prevention in pediatric respiratory clinics during the COVID-19 epidemic
}

\author{
Jing Zhang ${ }^{1} \cdot$ Lei Zhang $^{1} \cdot$ Yong Yin ${ }^{1} \cdot$ Quan Lu $^{2} \cdot$ Jian-Guo Hong ${ }^{3}$. Xiao-Bo Zhang ${ }^{4}$ Xiao-Jian Zhou ${ }^{3} \cdot$ Li-Bo Wang $^{4}$.

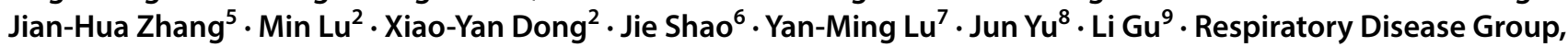 \\ Pediatric Committee of Shanghai Medical Association, Shanghai, China
}

Received: 29 February 2020 / Accepted: 8 June 2020 / Published online: 25 June 2020

(c) Children's Hospital, Zhejiang University School of Medicine 2020

\begin{abstract}
During the COVID-19 epidemic, it is important for ensuring infection prevention and control in the pediatric respiratory clinics. Herein, we introduced the practice of infection prevention and control in pediatric respiratory clinics in China. Triage measures for patients who visit respiratory clinics, quality control for pediatric respiratory clinics and other preventive measures for related examinations and treatment have been introduced in this review article.
\end{abstract}

\section{Introduction}

During the COVID-19 outbreak [1-3], pediatric respiratory clinics are still under normal service in China. Moreover, infection prevention has become a pressing issue in respiratory clinics for all pediatric hospitals. Therefore, we provide recommendations on best practice of infection

Yong Yin

yinyong9999@163.com

1 Department of Respiratory Medicine, Shanghai Children's Medical Center, Shanghai Jiao Tong University School of Medicine, No. 1678 Dongfang Road, Shanghai, China

2 Department of Respiratory Medicine, Children's Hospital of Shanghai Jiao Tong University, Shanghai, China

3 Department of Pediatrics, Shanghai General Hospital, Shanghai Jiao Tong University, Shanghai, China

4 Department of Respiratory Medicine, Children's Hospital of Fudan University, Shanghai, China

5 Department of Respiratory Medicine, XinHua Hospital, Shanghai Jiao Tong University School of Medicine, Shanghai, China

6 Department of Pediatrics, Ruijin Hospital, Shanghai Jiao Tong University School of Medicine, Shanghai, China

7 Department of Pediatrics, Renji Hospital, Shanghai Jiao Tong University School of Medicine, Shanghai, China

8 Department of Pediatrics, Shanghai FengXian District Central Hospital, Shanghai, China

9 Department of Pediatrics, Shanghai Tenth People's Hospital, Tong Ji University, Shanghai, China prevention in pediatric respiratory clinics in hospitals during the COVID-19 outbreak.

\section{Triage strategies in pediatric respiratory clinics}

During the severe acute respiratory syndrome (SARS) outbreak in China in 2003, outpatient triage system has been established and fever clinics have been set up in most hospitals [4]. Initial screening of patients can be done in the fever clinics for avoiding cross-infection in hospital; and the suspected cases can be quickly identified and triaged in the fever clinics. During the COVID-19 epidemic, any children suspected of SARS-CoV-2 infection will be managed at the fever clinics and should not be triaged to pediatric respiratory clinics, which can avoid cross-infection during in-hospital visits effectively $[4,5]$. Therefore, triage before respiratory clinic visits is very important. The triage flow is shown in Fig. 1. The workflow is as follows:

1. Set up striking signs at the entrance of the outpatient building and in all treatment areas to inform parents of children with fever and/or suspected symptoms about the hospital's triage flow and relevant inspection department route instructions.

2. Inform children and accompanying persons to wear masks. For those who do not have masks, the hospital needs to provide masks and instruct patients on proper 


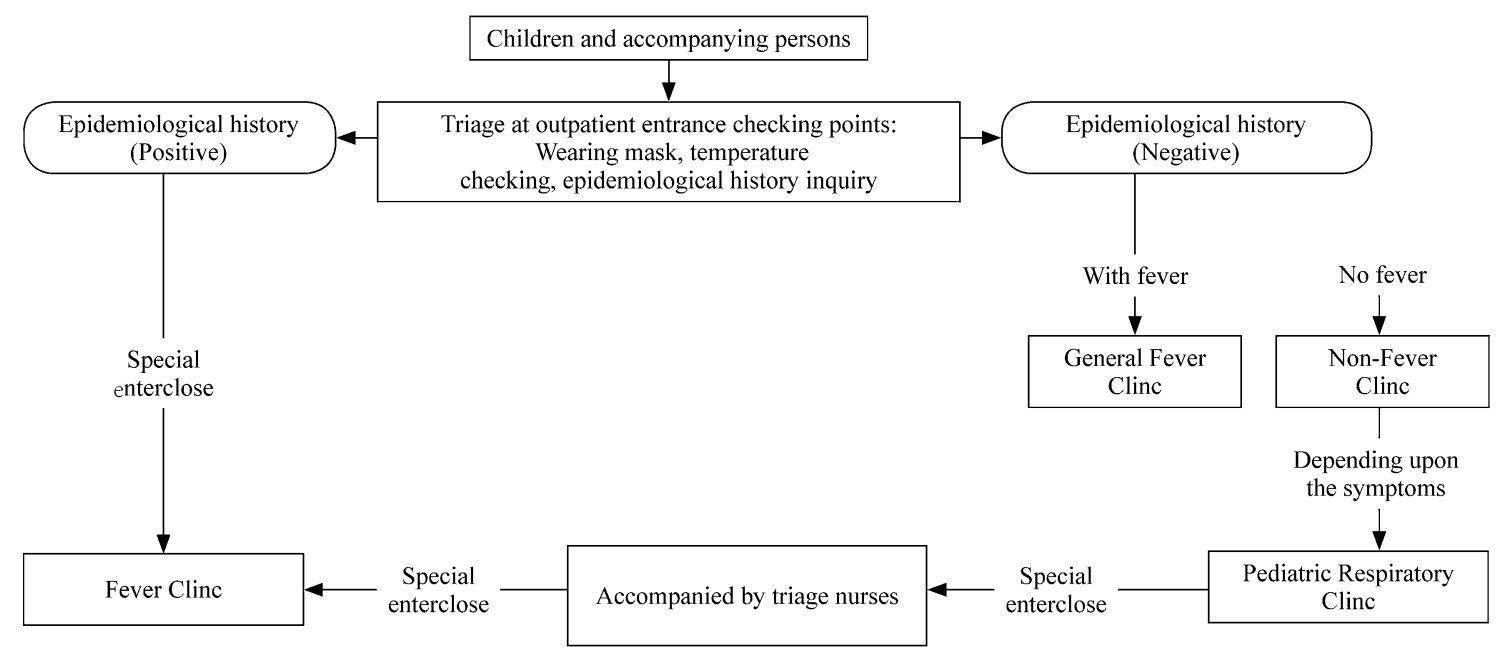

Fig. 1 Flowchart of the children's respiratory clinic triage procedure during the COVID-19 epidemic

wearing. All people should undertake the temperature checking at the entrance of the hospital.

3. Set up the outpatient triage area away from other areas, with a clear identification and good ventilation.

4. Conduct the initial triage during the outpatient visit. The triage staff should check the temperature of the children and the accompanying persons, and inquire about the epidemiological history. All the information collected for the patient should be recorded in the children's medical records. If the child with fever or respiratory symptoms has a suspicious epidemiological history, he/she should be isolated and guided to the fever clinic. At the second level of triage in the fever clinic, children should be divided into excluded cases and suspected cases, and visit different clinics located in different regions. Children who do not meet the criteria of suspected cases can be triaged to pediatric respiratory clinics as normal.

5. Medical workers who are responsible for triage should wear work clothes, medical surgical masks, disposable work caps, goggles/masks, medical isolation clothes, gloves, and medical protection masks (e.g., N95 masks).

\section{Quality control in pediatric respiratory clinics}

The main clinical manifestations of SARS-CoV-2 infection of children include fever, cough, and pharyngeal erythema. Fever and mild cough are common at disease onset for infected children. Compared with the adult cases, most children have milder manifestations $[3,6]$. The presentations of COVID-19 in children are also common manifestations due to other causes in respiratory clinic visitors. Although children who visit the pediatric respiratory clinics are patients who have been rigorously prescreened, there is a chance of omission. Therefore, it is very important for pediatric respiratory specialists to master the knowledge of clinical diagnosis and treatment of SARS-CoV-2 infection. To ensure quality control in pediatric respiratory clinics, there are some necessary tasks, such as training medical staffs, familiarizing staff with the COVID-19 diagnosis and treatment guidance, and ensuring adequate self-protection measures.

\section{Recognizing the clinical characteristics of COVID-19 in children [7]}

Compared with those in adults, most children have milder symptoms, a faster recovery, a shorter viral clearance time, and a better prognosis [8]. The clinical manifestations may be more insidious and difficult to distinguish than those caused by other pathogens. The contact history with infected patients and the epidemiological history of family clustering are very important in screening and diagnosing suspected cases. Although the incidence of critical pediatric cases is low, increased vigilance is especially needed for children with underlying diseases.

\section{Becoming familiar with updated diagnosis and treatment recommendations}

Pediatric respiratory specialists must be aware of the latest updates of diagnosis and treatment recommendations and know well about the characteristics of pediatric cases [7]. For pediatricians in non-designated hospitals, the clinical manifestations, diagnostic criteria, differential diagnosis, and case finding and reporting processes must be mastered. 
1. Medical and epidemiological histories

Pediatric respiratory specialists must be able to recognize suspected cases. The most important thing in recognizing suspected cases is the epidemiological history inquiry. The time frame for inquiry is within 14 days before the symptoms appear. The inquiry should include a travel and residence history focusing on epidemic areas, its surrounding areas and other communities with case reports; a contact history with a patient who had positive SARS-CoV-2 nucleic acid test results; a contact history with a patient who had fever or respiratory symptoms who had been to epidemic areas, its surrounding areas or communities with case reports; and cluster onset dates. Although prescreening should have already been performed, respiratory specialists still have to be highly vigilant and should ask about the epidemiological history once again.

The early symptoms in children are usually atypical. With the effective implementation of prevention and control measures, the number of imported cases gradually decreases, and the number of local cases increases. Some patients may not have a clear epidemiological history, which makes diagnosis complicated. Therefore, combined with the characteristics of pediatric cases, it is very important to identify clusters of infection and the infection status of family members. In addition to common respiratory symptoms, specialists also should pay attention to other symptoms, including fatigue, myalgia, dizziness, headache, and gastrointestinal symptoms, such as nausea, vomiting, and diarrhea. Moreover, some cases may not present with fever at all. Critical cases tend to show signs of shortness of breath, dyspnea, cyanosis, respiratory distress, septic shock, and multiple organ failure.

2. Laboratory and radiologic examinations

The main characteristics of infection in adults are a normal or decreased number of total white blood cells in the peripheral blood in the early stage, and a decrease in the lymphocyte count is a very common laboratory finding. However, in the existing pediatric cases, the total number of white blood cells is usually normal, and there are very few children with a decreased lymphocyte count; therefore, there are differences between children and adults. Some cases may exhibit a slight increase in C-reactive protein. SARS-CoV-2 nucleic acids can be detected in nasopharyngeal swabs, lower respiratory tract secretions, bronchoalveolar lavage fluid, plasma, and stool samples. SARS-CoV-2-infected patients, whether adults or children, present with varying changes in chest imaging during disease progression. Compared with the widespread and diffuse distribution of lesions in adults, children's chest radiographic lesions are relatively limited, with less diffuse distribution, and groundglass shadows are not as common as those in adults. Some shadows are pale cloud-like, and bronchial pneumonia is also common in children [9].

3. Diagnosis

Suspected cases must be diagnosed based on their epidemiological history, clinical symptoms, laboratory test results, and radiographic findings. Real-time fluorescent PCR detection of SARS-CoV-2 nucleic acids or viral gene sequences with high homology to known SARSCoV-2 can confirm infection. Early chest radiographic manifestations in pediatric cases are relatively limited, with patchy or ground-glass opacities distributed in the subpleural regions. With the disease progresses, the range of lesions expands, and ground-glass opacities and consolidation can coexist. In severe cases, computed tomography (CT) scans show bilateral diffuse lesions, mainly consolidation, and a few may appear as "white lung". We suggest that in the diagnosis, pediatricians should consider the epidemiological history and the nucleic acid test results, and high-resolution chest CT also should be taken into consideration. If it is difficult to judge a case in a pediatric respiratory clinic, it is recommended to quickly isolate the patient first and then consult experts at the hospital or at higher level hospitals for further guidance.

4. Differential diagnoses

Children are at risk of respiratory infections, especially in winter or during the alternation of the seasons. Mild COVID-19 cases need to be distinguished from upper respiratory tract infections caused by other viruses. Pneumonia needs to be distinguished from other infections caused by common pathogens in children, including influenza virus, adenovirus, respiratory syncytial virus, and Mycoplasma pneumoniae. Nasopharyngeal swabs or deep airway specimens can be used for routine pathogen detection in clinical cases, especially in suspected COVID-19 cases. Although there are few coinfections of SARS-CoV-2 and other pathogens in children, this phenomenon also needs attention from clinicians.

5. Case-finding and reporting

The main responsibility of the pediatric respiratory clinic is to identify suspected cases and not to overlook them. Once a suspected case is identified, the superior department of the hospital should be contacted. According to the procedures of the hospital, the suspected patients should be transferred to the fever clinic by specially assigned staff through a special passageway. The original clinic room and items inside the room need to be disinfected immediately. 


\section{Infection prevention and control in pediatric respiratory clinics}

\section{Infection prevention requirements for consulting room}

The consulting room should be mainly ventilated by openning windows or mechanical ventilation, and the room should be ventilated before the start of work. After work, the room should be ventilated for 15 minutes before disinfection. If necessary, the consulting room should be sterilized using ultraviolet light, hydrogen peroxide atomization or evaporation disinfection in strict accordance with the requirements of the management standards for hospital air purification (WS/t368-2012) [10]. The floor should be cleaned daily. After daily diagnosis and treatment activities, a disinfectant solution containing $500-1000 \mathrm{mg} / \mathrm{L}$ of effective chlorine can be used for wiping and disinfection at least once a day. In case of contamination, rooms should be cleaned and disinfected quickly. The surface of environmental objects can be wiped and disinfected with a hydrogen peroxide-wetted towel for $1 \mathrm{~min}$ or sprayed and wiped with a disinfectant solution containing $500-1000 \mathrm{mg} / \mathrm{L}$ of effective chlorine for at least 10 minutes. It is recommended to use hydrogen peroxide to spray or wipe the common medical supplies that need to be reused for disinfection for $1 \mathrm{~min}$. The diagnosis and treatment area should be equipped with fast-acting, hands-free disinfectant source that is recommended for disinfection.

\section{Protection for hospital staff}

Personal protective equipment for hospital staff includes work clothes, surgical masks, disposable work caps and work clothes, and gloves. Staff should wear protective masks, such as N95 respirators, because of their high risk of exposure. Consulting room should be equipped with special-purpose work clothes and a stethoscope. After use, they should be disinfected immediately, and they should not be removed from the consulting room to avoid cross-infection [11]. It is recommended that children and their families wear masks when entering the consulting room. If possible, masks should be prepared for children and their parents in the respiratory department.

\section{Requirements for pediatric respiratory specialists during diagnosis and treatment processes}

Epidemiological history taking and evaluation of clinical symptoms are once again emphasized and required for diagnosis or exclusion of suspected cases. During the whole diagnosis and treatment process, before and after direct contact with the patients, before an operation procedure, after contacting with blood or body fluids, and after direct contacting with the patient or their items, it is necessary to wash hands immediately or to use hand sanitizer gel that has a disinfection effect on virus.

\section{Long-term prescriptions, light inquiries, and online medical} services

Among long-term follow-up pediatric respiratory patients, a large proportion of children have asthma and other chronic lung diseases, such as bronchiolitis obliterans and bronchiectasis. For these specific populations, during the epidemic parents' greatest demand is to "maintain medication" while "going out as little as possible". For asthmatic children with good asthma control, the Shanghai Municipal Health Committee allowed medical prescription dosages to be appropriately extended to reduce the frequency of visits by children and parents.

At present, many hospitals in China have completed the development of patient follow-up and management platforms, which have also played an important role in disease follow-up during the epidemic. The Children's Hospital of Chongqing Medical University established an internet hospital. Through online hospitals, doctors can complete diagnoses, patients can stay indoors, prescriptions can be refilled online, medicine can be easily and quickly delivered to the home through the China Post. and doctors can "follow-up after diagnosis" via online platform. Online healthcare provided by hospitals has played an important role during the COVID-19 epidemic.

\section{Precautions for examinations and admission to the respiratory wards}

\section{Pulmonary function testing}

In the process of lung function testing, examinees need to repeatedly perform forced expiratory movements that often cause coughing and expectoration, which produce many droplets or aerosols, contaminating the detection instruments and indoor air. This results in a potential risk of contact and droplet transmission, leading to cross-infection during the SARS-CoV-2 epidemic. For those with suspected infection or in the acute phase of confirmed infection, it is suggested to suspend testing if the patient is not in an urgent need of clinical treatment. If the pulmonary function testing truly needs to be carried out, the specific prevention and control measures should be taken as follows. 
1. Prevention and control measures before testing

(1) Check the temperature before testing. It is recommended to use a hand-held noncontact thermometer. If the patient had a fever, the examination should be suspended.

(2) Epidemiological history taking information about travel/residence history in epidemic areas within the last 2 weeks, cluster onset dates or an epidemiologic association with someone infected with SARS-CoV-2 should be obtained. If the above epidemiological factors are positive, the examination should be suspended.

2. Infection prevention and control measures during testing

(1) The windows should be opened for natural ventilation. We also recommended to use an approved air purifier that is safe for humans and machines for air disinfection in the testing room. The air purifier should be regularly maintained, cleaned, and disinfected.

(2) A disposable, high-efficiency air filter (which can filter and absorb more than $99 \%$ of viruses and bacteria) must be used. Each subject should change their filter and avoid reuse.

(3) Pulmonary function testing staff should assess the operational risk and wear personal protective equipment in strict accordance with the different risk levels. The requirements include wearing work clothes, disposable work caps, goggles or protective screens, medical protective masks (such as N95 masks), wearing disposable latex gloves, and wearing disposable isolation clothes and disposable shoe covers when necessary. When medical protective masks, goggles and other protective articles are contaminated by saliva, sputum or other secretions, they should be replaced immediately.

3. Prevention and control measures after testing

(1) Hand washing and hand sanitizing.

(2) Clean and disinfect the sensors, respiratory equipment, instrument surfaces of pulmonary function testing instruments and the floor of the pulmonary function testing room in strict accordance with the technical specifications for the disinfection of medical institutions, and disinfect the air in accordance with the management specifications for air cleaning of the hospital.
(3) Dispose and manage medical waste generated during pulmonary function testing, such as disposable filters, in accordance with regulations for the disposal of medical waste and measures for the management of medical waste in medical and health institutions.

(4) Reasonably arrange the work of the staff in the pulmonary function test room to avoid fatigue; monitor their health in a timely manner, and monitor the temperatures and respiratory system symptoms of hospital staff.

\section{Bronchoscopy}

Bronchoscopy is an important procedure for the clinical diagnosis and treatment of children's respiratory diseases. While operating the bronchoscope, the medical staff must be in close contact with the patient. A patient's cough or forced respiration, which may produce a large number of droplets or aerosols, may contaminate equipment, indoor air, and personnel, and even splash onto the operator's cornea, skin, clothing, etc. These factors put the operator at a high risk of infection. During the epidemic period, the indications for bronchoscopy should be strictly limited. If the condition is not urgent, it is suggested to suspend the procedure. Specific prevention and control measures and requirements are as follows [12].

1. Prevention and control measures before bronchoscopy

Epidemiological history of the child and their parents should be evaluated to exclude possible suspected cases. Check the body temperature of the child and their parents with a hand-held, noncontact thermometer. If they have a fever, bronchoscopy should be postponed, and the fever screening process should be initiated. Children and their parents should wear protective surgical or medical masks (e.g., N95 masks). To reduce coughing in children, it is recommended to use intravenous anesthesia or general anesthesia rather than an inhaled or local surface anesthetic before surgery. Bronchoscopy should be carried out in an independent bronchoscope room with central air conditioning turned off and the windows opened for natural ventilation.

2. Operation requirements for bronchoscopy

For epidemic prevention and control, the operation should be carried out in an independent room for general patients without a contact history or related symptoms. The operator should be well protected according to the requirements of secondary protection, and the bronchoscopic examination should not be carried out without protective measures. Level II protective equipment including disposable working caps, protective medical masks (e.g., N95 masks), protective glasses or masks (antifog type), protective clothing or isolation clothing, 
disposable latex gloves, and disposable shoe covers should be worn.

3. Requirements after bronchoscopy

After bronchoscopy or contact with saliva, sputum and other secretions of patients, operators should wash their hands with flowing water and/or use quick-dry hand sanitizer for hand disinfection. In daily practical work, hand hygiene should be carried out in strict accordance with hand hygiene standards. Wearing gloves cannot replace hand hygiene. Hand hygiene should always be carried out after gloves are removed. Do not touch public materials after wearing gloves. After the completion of the bronchoscopy examination, all medical staff should replace their full set of protective equipment and strictly conduct hand hygiene before entering the general area.

In strict accordance with the technical specifications for the disinfection of medical institutions, clean and disinfect the bronchoscope, other relevant equipment, the examination bed and the ground with a chlorine-containing disinfectant. Precision instruments should be wiped with $75 \%$ alcohol. Air disinfection should be carried out in accordance with the management standard for hospital air purification. For example, ultraviolet room disinfection should be performed for more than 30 minutes. The rooms of suspected or confirmed patients should be disinfected after bronchoscopy examination. After finishing the disinfection process, the windows should be opened for ventilation. The medical waste generated during the bronchoscopy procedure, such as disposable biopsy forceps and sputum collectors, should be disposed of and managed in accordance with the relevant provisions of the regulations on the treatment of medical waste and measures for the management of medical waste in medical and health institutions.

\section{Hospitalization}

The epidemiological history of the children and accompanying parents must be confirmed again before admission, and suspected cases should never be admitted to the general ward. Children from fever clinics who have been excluded for SARS-CoV-2 infection can be admitted to the general ward after expert consultation. And they should be treated in a separate room in the ward.

\section{Coping strategies for special situations}

If the child is found to be suspected of SARS-CoV-2 infection at pediatric respiratory clinic visit, they should be reported to the supervisor and hospital management department immediately. Air disinfection should be strictly carried out in the examination room. The instruments should be cleaned and disinfected again. All the staffs who have contact with the confirmed infected children should be quarantined for medical observation according to the regulations.

Strengthen the supervision of infection control. For equipment parts that can be repeatedly disinfected and reused, such as respiratory pipes and valves, bacterial culture should be carried out regularly after disinfection, and the effective concentration of disinfectant used for immersion should be monitored to ensure the effectiveness of infection prevention and control measures.

During the epidemic, rapid screening, prevention, and control of SARS-CoV-2 infection have become the core focus. It is necessary to strengthen quality control management to isolate potential sources of infection, to cut off all routes of transmission, and to terminate cross-infection and transmission.

Author contributions All authors contributed equally to this paper. All authors drafted part of the manuscript and approved the final version.

Funding Health Science and Technology Project of Shanghai Pudong New Area Health and Health Committee (PW2017E-1), Science and Technology Support Project in Biomedical Field of "Science and Technology Innovation Action Plan" of Shanghai Science and Technology Commission (19441909000), and Health Science and Technology Project of Shanghai Pudong New Area Health and Health Committee (PW2019A-37).

\section{Compliance with ethical standards}

Ethical approval Not required for this review article.

Conflict of interest No financial or non-financial benefits have been received or will be received from any party related directly or indirectly to the subject of this article.

\section{References}

1. Chan JF, Yuan S, Kok KH, To KK, Chu H, Yang J, et al. A familial cluster of pneumonia associated with the 2019 novel coronavirus indicating person-to-person transmission: a study of a family cluster. Lancet. 2020. https://doi.org/10.1016/S0140 -6736(20)30154-9.

2. Dong Y, Mo X, Hu Y, Qi X, Jiang F, Jiang Z, et al. Epidemiological characteristics of 2143 pediatric patients with 2019 coronavirus disease in China. Pediatrics. 2020. https://doi.org/10.1542/ peds.2020-0702.

3. Lu X, Zhang L, Du H, Zhang J, Li YY, Qu J, et al. SARS-CoV-2 infection in children. N Engl J Med. 2020. https://doi.org/10.1056/ NEJMc2005073.

4. Zhang J, Zhou L, Yang Y, Peng W, Wang W, Chen X. Therapeutic and triage strategies for 2019 novel coronavirus disease in fever clinics. Lancet Respir Med. 2020;8:e11-2.

5. Zeng M, Zhai XW, Wang JS. 2019 novel coronavirus infection: pediatric professionals' perspectives and action. Chin J Infec Dis. 2020. https://doi.org/10.3760/cma.j.issn.1000-6680.2020.0003. (in Chinese) 
6. Cai J, Xu J, Lin D, Yang Z, Xu L, Qu Z, et al. A case series of children with 2019 novel coronavirus infection: clinical and epidemiological features. Clin Infect Dis. 2020. https://doi.org/10.1093/ $\mathrm{cid} / \mathrm{ciaa} 198$.

7. Shen K, Yang Y, Wang T, Zhao D, Jiang Y, Jin R, et al. Diagnosis, treatment, and prevention of 2019 novel coronavirus infection in children: experts' consensus statement. World J Pediatr. 2020. https://doi.org/10.1007/s12519-020-00343-7.

8. The Society of Pediatrics of Chinese Medical Association, the Editorial Board of China Journal of Pediatrics. Recommendations for the diagnosis, prevention and control of the 2019 novel coronavirus infection in children. Chin J Pediatr. 2020. https://doi. org/10.3760/cma.j.issn.0578-1310.2020.0004.

9. Li W, Cui H, Li K, Fang Y, Li S. Chest computed tomography in children with COVID-19 respiratory infection. Pediatr Radiol. 2020. https://doi.org/10.1007/s00247-020-04656-7.

10. National Health Commission of the People's Republic of China. Management specification of air cleaning technique in hospitals
(WS/T 368-2012). https://www.nhc.gov.cn/wjw/s9496/20120 4/54511.shtml. Accessed 26 Feb 2020.

11. National Health Commission of the People's Republic of China. Notice of the General Office of the National Health Commission on the issuance of guidelines on the scope of use of common medical protective supplies in the prevention and control of pneumonia with new coronary virus infection (trial). https://www.nhc. gov.cn/yzygj/s7659/202001/e71c5de925a64eafbe1ce790debab5 c6.shtml. Accessed 9 Apr 2020.

12. Group of Interventional Respiratory Medicine of Chinese Thoracic Society. Expert consensus for bronchoscopy during the epidemic of. Novel Coronavirus infection (Trial version). Chin J Tuberc Respir Dis. 2020. https://doi.org/10.3760/cma.j.i ssn.1001-0939.2020.0006. (in Chinese)

Publisher's Note Springer Nature remains neutral with regard to jurisdictional claims in published maps and institutional affiliations. 